\title{
La langue de Wall Street entre le milieu professionnel et le grand public
}

\section{Michel Van der Yeught}

\section{(2) OpenEdition}

1 Journals

\section{Édition électronique}

URL : http://journals.openedition.org/asp/995

DOI : $10.4000 /$ asp.995

ISBN : 978-2-8218-0396-1

ISSN : 2108-6354

\section{Éditeur}

Groupe d'étude et de recherche en anglais de spécialité

Édition imprimée

Date de publication : 1 mars 2004

Pagination : 23-36

ISSN : 1246-8185

Référence électronique

Michel Van der Yeught, "La langue de Wall Street entre le milieu professionnel et le grand public », ASp [En ligne], 43-44 | 2004, mis en ligne le 13 mars 2010, consulté le 19 avril 2019. URL : http:// journals.openedition.org/asp/995 ; DOI : 10.4000/asp.995

Ce document a été généré automatiquement le 19 avril 2019

Tous droits réservés 


\title{
La langue de Wall Street entre le milieu professionnel et le grand public
}

\author{
Michel Van der Yeught
}

\section{Introduction}

1 Cet article traite de la langue des professionnels de Wall Street. Par là, il faut entendre les métiers impliqués dans le commerce des valeurs mobilières sur les marchés boursiers aux États-Unis. Figurent parmi ces professionnels les courtiers (brokers) qui opèrent en tant qu'agents ou intermédiaires entre deux partis; les négociants en titres (traders) qui interviennent pour leur propre compte; les spécialistes (specialists) et les teneurs de marché (market makers) qui agissent en tant qu'agents ou en tant que négociants tout en assumant des responsabilités particulières sur les marchés; les banquiers d'affaires ( investment bankers) qui organisent l'émission de nouveaux titres; enfin, les analystes qui évaluent les performances des valeurs.

La définition de ces professions par le qualifiant de «Wall Street » exige d'être précisée. Wall Street est l'une des plus anciennes rues de New York. Dès la fin du XVII ${ }^{e}$ siècle, les activités commerciales puis financières s'y concentrent et s'y développent au XIXe siècle pour constituer au $\mathrm{XX}^{\mathrm{e}}$ siècle le plus grand centre financier du monde. L'appellation «Wall Street» correspond actuellement à quatre définitions distinctes. Premièrement, Wall Street désigne la rue elle-même tracée sur l'emplacement d'un ancien mur qui protégeait la ville d'origine (New Amsterdam) des agressions venant de Manhattan (Levison 1961: 26-36). Deuxièmement, par un effet de synecdoque particularisante (le tout exprimant la partie), elle désigne l'institution la plus importante qui y est installée, la première bourse de New York ou New York Stock Exchange (NYSE). Troisièmement et quatrièmement, par des synecdoques de plus en plus généralisantes (la partie exprimant le tout), Wall Street désigne le quartier financier de New York, et, au-delà, la finance américaine en général. La langue de Wall Street, qui émane de la langue naturelle appelée 
anglais, est donc employée par l'ensemble du milieu boursier américain. L'expression rappelle cependant que cette langue a été et reste puissamment influencée par l'activité de la bourse de New York et particulièrement par celle des professionnels qui y opèrent. C'est en effet sur le parquet du NYSE, plus que partout ailleurs depuis deux siècles, que la langue de Wall Street se crée et se renouvelle.

Étudier la langue de Wall Street nous amène à nous poser deux questions. Premièrement, cette langue comporte-t-elle une terminologie, c'est-à-dire un ensemble de notions et de termes nécessaires à l'activité boursière ? Pour répondre à la question, il est nécessaire de soumettre le lexique spécialisé de cette langue aux exigences de la terminologie. Celles-ci ont été profondément marquées par les théories d'Eugène Wüster et du Cercle de Vienne qu'il dirigeait (Van Campenhoudt 1997: 2). Dans le projet wüsterien, la science terminologique doit contribuer à la structuration et à l'unification des connaissances grâce à la mise en système de termes rigoureusement définis, classés et hiérarchisés les uns par rapport aux autres. Bien que le projet wüsterien soit actuellement réévalué, un examen de la langue de Wall Street par rapport à ses exigences est un préalable indispensable à son étude. Nous consacrerons notre première partie à cet éclairage.

La deuxième question concerne les relations que la langue des professionnels de Wall Street entretient avec la population américaine. En effet, au-delà de sa relation étroite avec la bourse, la langue de Wall Street a une facette publique. La bourse ne fonctionne pas en vase clos. Elle travaille pour des dizaines de millions d'investisseurs non professionnels. En 1999, une étude concluait que 79 millions d'Américains détenaient directement ou indirectement des actions (Levitt 2002: 245). Or, la perception que le grand public a de la langue de Wall Street est remarquablement constante depuis plus d'un siècle. Cette langue est considérée comme énigmatique, impénétrable et incompréhensible aux non-initiés. Des appellations généralement péjoratives abondent pour la caractériser: Wall Street slang, Wall Street jargon, Wall Street speak, Wall Street parlance, Wall street lingo. Un observateur du XIX siècle la caractérisait déjà comme " $a$ dialect which is caviare to the multitude » (Medbury 1968 : 132). L'analyse de cette langue de professionnels ne peut ignorer ce qu'en pensent leurs clients, même s'ils expriment leur perception de façon subjective. Notre objectif est d'expliquer du point de vue linguistique pourquoi la langue de Wall Street est perçue comme un jargon et/ou un argot désagréablement énigmatique par le grand public. Cette question nous amènera à considérer la langue de Wall Street comme un double moyen de communication: le premier, interne au milieu professionnel; le deuxième, externe, du milieu professionnel vers le grand public. Nous consacrerons nos deuxième et troisième parties à ces deux aspects.

\section{La langue de Wall Street comporte-t-elle une terminologie?}

5 L'un des critères les plus importants de la terminologie scientifique est la motivation des termes. Pierre Guiraud rappelle que «les langues scientifiques se caractérisent par la motivation de leur vocabulaire » (Guiraud $1978: 5-6$, in Setti $2000: 163$ ). Un terme désigne une notion qui fait l'objet d'un accord préalable de la part des spécialistes. La démarche qui part de la notion pour aboutir au terme est qualifiée d'onomasiologique, par opposition à la démarche sémasiologique qui part du terme pour aboutir à la notion. Le terme, quoique conventionnel, est motivé lorsqu'il est monosémique et défini en relation 
avec d'autres termes par rapport auxquels il est classé et hiérarchisé au sein d'un système cohérent (Setti 2000 : 14). Grâce à cette motivation, la langue de spécialité peut tendre, comme le dit Pierre Lerat, vers la prévisibilité maximale (Lerat 1997 : 7). Ainsi, à partir du terme stock (action), un locuteur natif de l'anglais pourra prévoir sans trop d'erreur que la notion «action-qui-bénéficie-d'avantages-préférentiels » sera désignée par le terme preferred stock; que la notion «action-qui-donne-le-droit-de-voter" pourra s'appeler voting stock; et que par conséquent la notion "action-qui-ne-donne-pas-le-droit-devoter » s'appellera non-voting stock. Le terme motivé est dénotatif et non connotatif. Il est rigoureusement défini en lui-même par un certain nombre de caractères qui éliminent les ambiguités et les synonymes stricts. Il doit pouvoir être normalisé au niveau international par des normes de type ISO. Parce que les systèmes terminologiques de type wüsterien sont indépendants des langues naturelles, les termes sont généralement symétriques d'une langue à l'autre (Setti 2000: 169), c'est-à-dire qu'ils sont monoréférentiels quelle que soit la langue considérée. Par conséquent, ils peuvent être traduits sans distortion de sens. Au contraire, certains facteurs fragilisent la relation entre la notion et le terme et contribuent à le démotiver. La démotivation entraîne des phénomènes de polysémie, de synonymie ou d'asymétrie qui nuisent à l'univocité et donc à la précision du terme (Setti $2000: 163-170$ ).

Cela posé, ce qui frappe dans le lexique de Wall Street, c'est la grande hétérogénéité de la motivation de ses termes. D'un côté, un terme comme beta apparaît fortement motivé, de l'autre, un terme comme blue chip apparaît démotivé.

7 Le beta désigne la sensibilité des fluctuations d'une valeur à celle d'un indice (Jacquillat et Solnik 1989 : 94). Autrement dit, c'est le coefficient qui mesure la volatilité d'un titre boursier. Cette mesure est largement utilisée par les professionnels de la bourse, particulièrement par les gestionnaires de portefeuille, les chartistes, les courtiers, les analystes et les spéculateurs. La notion de coefficient de volatilité correspond bien à la définition de ce qu'est une notion par la norme ISO 1087 de 1990: "C'est une unité de pensée constituée par abstraction à partir des propriétés communes à un ensemble d'objets » (ISO 10871990 : 1, in Van Campenhoudt 1997 : 2). Dans ce cas, l'unité de pensée abstraite est présentée sous la forme d'une formule mathématique. Le terme beta est conventionnel et a été adopté par l'ensemble des spécialistes de la profession après accord sur la notion. La démarche d'élaboration du terme est donc onomasiologique. La relation terme-notion est strictement monoréférentielle dans le domaine boursier et elle n'est pas fragilisée par d'éventuels synonymes. Le terme est donc monosémique et purement dénotatif. En outre, il peut faire l'objet d'une normalisation de type ISO et donner lieu à des classifications en devenant hypéronyme d'une série de betas hyponymes auxquels sont ajoutés des caractères supplémentaires (Lerat $1990: 81-83)$ : par exemple le market beta, le portfolio beta, l'index beta, et divers historical betas. De même, les betas peuvent entrer dans une classe plus large, appelée par exemple «indicateurs d'évolution ", dans laquelle figureront également les coefficients alpha et delta, termes qui possèdent les mêmes caractéristiques terminologiques que le beta. Enfin, le terme beta ayant été choisi dans l'alphabet grec en dehors de la langue naturelle de l'anglais, il s'abstrait des différentiations nationales et il s'inscrit dans un vocabulaire à vocation universelle (Guilbert 1973: 12). Il est donc extrêmement symétrique d'une langue à l'autre et se traduit par "bêta", ou «beta " sans accent circonflexe, dans toutes les langues naturelles qui contiennent un lexique spécialisé des professionnels de la bourse. Il se prête ainsi parfaitement à la traduction automatique. 
8 À l'inverse, le terme blue chip présente toutes les caractéristiques de la démotivation. Dans le lexique de Wall Street, blue chip désigne les valeurs de premier ordre, mais le terme ne fait pas référence à une notion clairement définie dans un domaine de connaissances extérieur à celui de la bourse. L'approche onomasiologique est donc impraticable. Le terme semble appartenir à la classe des stocks (actions en anglais américain), des shares (actions en anglais britannique) ou des securities (titres ou valeurs), mais les lexèmes blue et chip ne renvoient à aucun étiquetage évoquant ces classes de notions. La prévisibilité du terme est donc très faible et elle se trouve réduite encore davantage par le fait que dans la compétence d'un locuteur de langue naturelle, les deux sèmes évoquent des notions totalement étrangères à la bourse. Leur combinaison, blue + chip, renvoie au monde des casinos où, sur les tables de jeu, les jetons bleus (blue chips) ont la valeur la plus élevée par rapport aux jetons rouges et blancs. Par métaphore, le terme désigne donc les valeurs des entreprises les plus sérieuses et les plus rentables par opposition aux valeurs risquées. Il faut donc partir du terme pour retrouver la notion (approche sémasiologique). Dans le lexique de Wall Street, blue chip appartient à un groupe lexical défini sans précision qui comprend des éléments lexicaux construits de façon similaire. Ainsi, le terme red chips désigne les titres de sociétés chinoises cotées à Hong-Kong, le rouge évoquant la Chine communiste. Black chips désigne les titres d'entreprises sud-africaines, le noir évoquant dans ce cas la race noire du continent africain. Green chips désigne les titres d'entreprises soucieuses d'écologie. Il est pourtant clair que cette classification ne satisfait pas aux exigences de la terminologie, les différentes couleurs qui caractérisent les chips ne correspondant à aucune définition précise. Enfin, blue chip est très peu symétrique d'une langue à l'autre et donc très difficile à traduire. Il est également pratiquement impossible à normaliser.

9 Au sein de cette même langue spécialisée, le lexique présente donc une importante hétérogénéité de motivation terminologique. Pour la mettre en évidence, il est possible de tracer un axe de la motivation des éléments lexicaux allant de la motivation la plus forte à la plus faible. Sur cet axe, on peut construire un tableau répartissant le lexique en fonction de la motivation de ses éléments (voir en Annexe le Tableau du spectre terminologique de la langue de Wall Street). Quoique l'axe constitue un continuum, une répartition en plusieurs groupes facilite l'analyse. Nous pouvons placer dans le groupe 1 les éléments les plus motivés (par exemple, beta, alpha, delta, PER) et dans le groupe 5 les éléments les moins motivés (par exemple, triple witching hour, blue-sky laws, red herring). Entre ces deux pôles, le terme stock que nous analysons comme fortement motivé figure dans le groupe 2 car il présente une motivation moins forte que beta. De même, les éléments du groupe 4 sont faiblement motivés, mais par rapport au groupe 5 ils gardent généralement des caractères de prévisibilité supérieurs qui permettent d'approcher leur signification.

10 Le tableau présente ce qui pourrait être appelé le spectre terminologique de la langue de Wall Street. Il montre que les différents critères discriminant de la terminologie évoluent de façon cohérente en fonction de la motivation. En effet, quand celle-ci est forte, la symétrie, la capacité de classification et de normalisation sont fortes. Au contraire, au fur et à mesure qu'elle s'affaiblit, ces trois critères s'affaiblissent d'autant. D'après notre analyse, les seules caractéristiques qui restent constantes sur la totalité de l'axe sont la monosémie et la dénotation. Même s'ils sont totalement démotivés (comme dans le groupe 5), les termes ne sont pas ambigus et ne sont pas cause d'erreurs entre professionnels. 
11 Pour expliquer l'hétérogénéité du lexique wallstreetien, il faut analyser la fonction et l'origine des termes. Le lexique des groupes 1 et 2, par exemple, est surtout utilisé par les analystes, les gestionnaires de portefeuille, les banquiers d'affaires. Ce sont des métiers d'évaluation, de mesure, de conception, de prise de décision. Ils ont besoin d'une terminologie analytique précise. Les termes motivés participent de la culture de l'écrit. Ils sont issus de domaines de connaissance extérieurs à Wall Street et scientifiquement structurés: l'économie, les mathématiques financières, la gestion ou la finance d'entreprise. Souvent élaborés par des universitaires étrangers à Wall Street, ils satisfont aux exigences terminologiques de la communauté scientifique. Le beta mentionné plus haut fut conçu en 1952 à l'université de Chicago par l'Américain Harry Markowitz, et amélioré par William Sharpe de UCLA en 1964 (Bernstein 1992 : 41-60, 75-88). Les métiers qui font usage de ce lexique motivé se sont développés assez récemment dans la communauté boursière, à partir des années 1970 (Bernstein 1992 : 2-14). Dans la langue de Wall Street, la terminologie scientifiquement motivée est donc historiquement récente et s'appuie sur un système référentiel de notions extérieures à Wall Street. C'est pour cette raison que l'approche onomasiologique lui est applicable.

12 À l'autre extrémité du spectre en revanche, le lexique démotivé des groupes 3, 4, 5 est largement issu des salles de marché et de l'anglais parlé. Il ne s'agit pas du lexique d'une langue d'analyse mais bien plutôt de celui d'une langue d'action dont la fonction principale est l'exécution correcte et rapide des transactions. Cette langue doit être non ambiguë, mais elle ne renvoie pas pour autant à un système de connaissances extérieur. En réalité, son seul critère de motivation stable réside dans le fait que les membres de la communauté professionnelle la comprennent et l'utilisent. Dans cette partie du spectre, la motivation sémantique n'est pas une motivation référentielle, c'est une motivation d'usage, et souvent d'usage oral. La seule garantie de la signification de ces termes se trouve dans la communauté professionnelle. Pour que la monosémie reste satisfaisante sur toute la longueur de l'axe, il est donc nécessaire que motivation terminologique et motivation d'usage soient inversement proportionnelles. Le lexique de Wall Street est bien monosémique et les boursiers se comprennent parfaitement parce que lorsque la motivation terminologique est faible, la motivation d'usage est forte, et inversement. L'exemple de blue-sky laws illustre parfaitement la force de la motivation d'usage. Ce terme, extrêmement démotivé du point de vue terminologique, désigne les textes légaux adoptés par certains États américains, notamment dans l'Ouest, pour réglementer les émissions de titres boursiers. Les professionnels eux-mêmes ignorent la provenance précise du terme. Certains situent son origine dans un discours prononcé par un juriste du Kansas vers 1900 (Pessin 1988 : 35). Quoi qu'il en soit, l'usage (oral à l'origine, notonsle) a prévalu dans la communauté boursière, et aucun terme mieux motivé et plus prévisible, tel que «state securities laws», n'est venu le remplacer. La même démonstration pourrait être menée pour de nombreux autres termes du groupe 5 comme red herring ou pink sheets qui sont profondément enracinés dans la motivation d'usage et qui sont néanmoins devenus des termes officiels de la profession (Shook \& Shook 1990 : $309,344)$. Cette partie non motivée du lexique de Wall Street contient à la fois des éléments lexicaux qui remontent au XVIII ${ }^{\mathrm{e}}$ siècle comme bull et bear (Zweig: 32) et d'autres beaucoup plus récents (triple witching hour date des années 1970). Néanmoins, la motivation d'usage est le mode d'élaboration des termes le plus ancien. Historiquement, c'est ainsi que la langue des marchés boursiers américains s'est constituée. 
13 Il y a donc bien une terminologie wallstreetienne, mais elle est marquée par une grande hétérogénéité qui résulte de deux forces opposées. D'un côté, les exigences de la terminologie moderne suscitent l'apparition de termes nouveaux scientifiquement motivés qui renvoient à des notions reliées entre elles dans des systèmes de connaissance structurés. En sens opposé, la communauté professionnelle de Wall Street perpétue et renouvelle un lexique traditionnel, scientifiquement démotivé, qui apparaît sur le long terme comme la langue de Wall Street authentique et originelle.

Lorsque Pierre Lerat explique " qu'une langue spécialisée est d'abord une langue écrite et qu'elle est au service d'une fonction majeure : la transmission des connaissances » (Lerat 1995 : 21), la langue traditionnelle de Wall Street apparaît comme une anomalie. Elle est largement d'origine orale, même parfois gestuelle, et sa démotivation, liée à son caractère imprévisible, la rend peu apte à transmettre des connaissances. De fait, de nombreux témoignages attestent qu'elle a plutôt pour fonction d'en opacifier la transmission. Un observateur du XIXe siècle note que beaucoup de gens souhaitent maitriser la langue de Wall Street, mais que malgré leurs efforts ils ne réussissent qu'à se ridiculiser en donnant des ordres contraires à leurs intentions (Medbury 1968: 200). Il explique qu'il est impossible d'apprendre cette langue en dehors du parquet et que les professionnels parviennent toujours à repérer les amateurs grâce à un subtle something, un petit détail linguistique qui détonne et qui les trahit (Medbury 1968: 132). De fait, de nombreuses anecdotes attestent que les professionnels utilisent des termes motivés par le seul usage pour marquer la différence entre ceux qui le connaissent et ceux qui l'ignorent. Par exemple, la chronique rapporte de nombreuses plaisanteries où des anciens demandent à un jeune grouillot (un garçon de course du parquet) d'apporter des courriers à Mr. Bull (littéralement, M. Haussier, c'est-à-dire quelqu'un qui escompte une hausse des cours) ou à Mr. Bear (littéralement, M. Baissier, c'est-à-dire quelqu'un qui escompte une baisse des cours) (Odean 1988 : 147). Dans ce cas, la langue de spécialité n'a pas pour fonction de transmettre un message le plus efficacement possible, elle sert à identifier les initiés qui en maîtrisent le code et à exclure les non-initiés.

Dans cette perspective, certains éléments lexicaux apparaissent comme de véritables labyrinthes sémantiques où, seule, la communauté permet de se repérer. L'exemple de Clara Bow illustre bien cette fonction à la fois opacifiante et initiatique du lexique. Pendant des années, les titres de la société désormais dissoute ITT (International Telegraph \& Telephone) ont été appelés Clara Bows par les traders. En voici la raison. Le code boursier d'ITT était IT. En 1927, Hollywood produisit un film intitulé It. La vedette en était l'actrice Clara Bow, devenue célèbre sous nom de «the It girl ». D'où le nom de Clara Bow donné par les traders au code IT (Odean 1988 : 27). Dans un article de 1997, P. Lerat écrit que, dans les langues spécialisées, « la langue ne fait que mettre de l'ordre dans la connaissance des choses" (Lerat 1997: 3). L'exemple de Clara Bow nous amène à paraphraser P. Lerat, mais à l'envers. Par sa langue, la communauté professionnelle de Wall Street s'ingénie à semer le désordre dans la connaissance. Elle secrète des éléments lexicaux qui, au-delà de leur fonction opératoire, servent de "marqueurs d'initiation ». Ils permettent de repérer ceux qui appartiennent au métier et qui savent en décrypter le code linguistique.

L'efficacité de ces "marqueurs d'initiation » est accrue par leur renouvellement rapide (Odean 1988: 1). Certains d'entre eux sont des classiques comme bull et bear, mais le lexique des traders s'enrichit tous les jours de nouvelles expressions tandis que d'autres sont rapidement frappées d'obsolescence. Ce faisant, la langue entretient en permanence 
son opacité et elle empêche les non-initiés de pouvoir l'apprendre. C'est là l'origine de la perception populaire de la langue de Wall Street. Elle se présente comme un jargon ou un argot expressément conçu pour exclure ceux qui n'en connaissent pas le code et pour entretenir les privilèges de ceux qui le maîtrisent.

Ce dernier développement nous autorise à formuler l'hypothèse suivante. Si la langue de Wall Street s'est construite, non seulement en fonction des exigences techniques de sa spécialité, mais encore sur l'opposition initiés/non-initiés linguistiques, il est probable qu'elle présentera des caractéristiques sensiblement différentes selon qu'elle est employée entre professionnels ou pour communiquer avec le grand public.

\section{La langue de Wall Street utilisée entre professionnels}

18 Wall Street représente le marché boursier (stock market) et la langue spécialisée que ses professionnels utilisent entre eux est donc une langue de marché et de transactions. Elle est directe et peu conceptuelle afin de faciliter des échanges oraux extrêmement rapides. Elle s'enracine dans une langue populaire, quotidienne et imagée. Avoir des titres se dit to be long, ne pas les avoir se dit to be short. Une option de vente est " proposée » à l'achat, c'est un put. Une option d'achat est " appelée ", c'est un call. Jusqu'à il y a une trentaine d'années, cette langue orale a servi de base aux échanges selon la devise de la Bourse de Londres pratiquée aussi à New York : « My word is my bond».

19 Cependant, la bourse a une particularité par rapport aux autres types de marchés: les intervenants ne voient jamais les produits qu'ils y échangent. Pour leur donner une réalité tangible et les rendre attractifs, la langue de Wall Street fait largement appel aux figures de style, et particulièrement aux métaphores. Quand Boileau et Dumarsais, cités par Paul Ricœur (Ricœur 1975: 85) disent qu'« on trouve plus de métaphores sur les marchés qu'à l'Académie française", ils ont a fortiori raison en ce qui concerne les marchés boursiers dont la langue est largement métaphorique. La définition de la métaphore par Lakoff et Johnson " the essence of metaphor is understanding and experiencing one kind of thing in terms of another » (Lakoff \& Johnson $1980: 5$ ) s'applique parfaitement ici. Les entreprises dévaluées sont des anges déchus (fallen angels) ; le trader qui fait des aller et retour rapides sur le marché fait du scalping; les glamour stocks ou les go-go stocks attirent autant les convoitises que les glamour girls ou que les go-go girls. Certaines de ces métaphores se sont figées et sont devenues de véritables termes comme blue chip et junk bond. D'autres sont filées et entremêlées les unes aux autres et constituent des champs métaphoriques complexes qui structurent la perception des phénomènes financiers. Ainsi dans le cadre d'un raid boursier, une métaphore médiévale filée fait de l'entreprise attaquée une sleeping beauty agressées par un black knight (le raider) et secourue par un white knight, qui tous deux se disputent ses crown jewels (les meilleures divisions de la société en question) (Odean 1988 : 105-106, 114). Dans leur ouvrage Metaphors We Live By, Lakoff \& Johnson (1980) dépassent la conception aristotélicienne de la métaphore effet de style. Ils pensent que les concepts eux-mêmes sont métaphoriquement structurés et que les processus mentaux sont largement métaphoriques. Leur analyse correspond parfaitement au fonctionnement de la langue que les professionnels de Wall Street utilisent entre eux. Autant la terminologie motivée des groupes 1 et 2 de notre tableau se révèle wüsterienne, autant la langue des marchés est "lakoffienne» et perçoit 
principalement la réalité à travers des métaphores. Certaines d'entre elles sortent du cadre de Wall Street et se répandent dans le grand public par l'intermédiaire de la presse boursière. Catherine Resche (1995) a montré qu'elles forment des réseaux convergents en anglais et en français et qu'elles atteignent ainsi un certain degré de symétrie d'une langue à l'autre.

Les métaphores sont également révélatrices de la communauté qui les emploie. La langue de Wall Street est réputée pour être crue et humoristique. Elle abonde en analogies amusantes et en métaphores violentes, sexuelles ou scatologiques qui alimentent la longue tradition des Wall Street jokes (Wall Street Joke Book 1997 : VII). Ces plaisanteries, qui oscillent entre les blagues de collégiens immatures et les obscénités de corps de garde, sont typiques d'une communauté largement masculine. Elles contribuent à faire de ce jargon un argot vulgaire perçu par le public comme un moyen d'expression d'un niveau inférieur. Dans un environnement professionnel marqué par une grande tension nerveuse, cet argot joue le rôle d'un exutoire au stress car il laisse libre cours aux pulsions profondes des traders. Il provoque une détente humoristique par la libération des inhibitions selon des mécanismes psychologiques semblables à ceux que Freud a étudiés dans Le mot d'esprit et ses rapports avec l'inconscient (1930: 222-239). Un bon exemple de ce phénomène est la sémantisation et la métaphorisation des sociétés cotées. Les boursiers affublent les actions des compagnies de surnoms qui facilitent leur identification et qui suscitent le rire. Certains sont connus du grand public comme «Big Blue » pour IBM ou "Big Yellow » pour Kodak. Ils peuvent être anodins comme « Mickey » pour Walt Disney, ou «Burgers» pour McDonald's; ou encore moqueurs comme "Mad Dog» pour McDonnell Douglas ou «Hot Beds» pour Holiday Inn. Les traders métaphorisent également souvent les codes boursiers. Chaque société cotée reçoit un identifiant de une à trois lettres que les opérateurs sémantisent à loisir. Les résultats, souvent comiques mais parfois orduriers, sortent rarement du quartier financier. Si le code BA de Boeing Aircraft reste dans les limites de la décence en devenant «Bare Ass », les traders n'ont pas hésité à surnommer « Fuck Me » l'infortunée Fairbanks Morse Company, code FKM (Odean 1988 : 44). Dans ces conditions, on mesure à quel point certaines métaphores ou certaines unités phraséologiques peuvent être difficiles à utiliser par les femmes traders. L'argot de Wall Street ne filtre pas que les non-initiés, il constitue également un obstacle subtil à la féminisation des professions boursières. Alice Jarcho, la première femme trader n'a été autorisée à travailler sur le parquet qu'en octobre 1976 et elle n'a obtenu son accréditation que de haute lutte (Colbert 2001 : 182).

21 La sémantisation des codes boursiers est un phénomène linguistique significatif parce qu'il corrobore l'analyse menée plus haut. Les codes présentent, en effet, toutes les caractéristiques de termes fortement motivés et leur sens est ouvert à tous ceux qui veulent les apprendre. Ils satisfont à la tendance wüsterienne et universaliste de la terminologie scientifique de Wall Street et méritent de figurer dans le groupe $1 \mathrm{du}$ tableau. En interne cependant, la communauté professionnelle reprend ses droits. En métaphorisant les codes, elle se les réapproprie et elle les crypte en un langage accessible aux seuls initiés, langage dont les termes doivent figurer dans les groupes 3,4 ou 5 . Les codes boursiers sémantisés illustrent parfaitement la tendance lakoffienne de l'argot de Wall Street.

22 Les métaphores de la langue de Wall Street ont intéressé de nombreux chercheurs. Dans le sillage de Lakoff et Johnson, ils ont essayé d'y délimiter des champs métaphoriques et de découvrir à partir de quelles mégamétaphores profondes ils se constituent. Analysant 
la presse boursière, Catherine Resche isole quatre grands registres : la météorologie, la marine, les sports, la santé (Resche 1995 : 95-97). De son côté Alan Partington, constatant la fréquence des métaphores relevant de la course, propose comme mégamétaphore : THE STOCK MARKET IS A RACE ${ }^{1}$ (Partington 1995: 32). Pour ma part, j'observe une convergence frappante entre trois éléments complémentaires : une phraséologie de la violence particulièrement fournie (to hammer the market, to squeeze the shorts); de très nombreuses hyperboles exprimant la domination par la force ou par la ruse (Icahn the Terrible, the Ulysses of the stock market, the Wizard of Wall Street); enfin, un bestiaire métaphorique très développé. Les animaux peuplent l'argot de Wall Street presque autant que les fables de La Fontaine, et ils y jouent un rôle assez semblable. Dans son ouvrage sur «la fabrique des fables» de La Fontaine, Patrick Dandrey (Dandrey 1996: 245-246) explique que le fabuliste a recours aux animaux parce qu'ils expriment sans fard leur condition et leurs appétits et qu'ils sont donc très propres à figurer les passions humaines les plus violentes. De la même manière, les raiders de Wall Street attaquent des sociétés, les vulture investors se repaissent de titres sous-évalués en attendant la hausse, tandis que le herd instinct pousse les sheep, les mullets ou les lambs à se faire massacrer ou tondre par les loups du marché (Elias 1971). Dans le même style inquiétant, des traders contemporains comparent des stratégies boursières qui tournent à la catastrophe au fait d'être "eaten alive by the market» (Niederhoffer 1997: 353; Sperandeo 1993: 15). Ces différentes figures de style expriment la concurrence extrême qui règne entre les acteurs de Wall Street et convergent à mon avis vers une mégamétaphore majeure : THE MARKET IS A JUNGLE (Ney 1970; Sobel 1970 : 83). En effet, cette métaphore darwinienne englobe de nombreuses autres métaphores, comme celles du sport ou celle du sexe essentiellement perçues comme violence et comme domination, qui apparaissent comme les facettes secondaires de ce message profond: la loi du marché, comme la loi de la jungle, est la lutte pour la survie. Rappelons que les théories de Darwin ont été très bien accueillies aux États-Unis (Hofstadter 1959: 5), et que dès 1870, seulement onze ans après la publication de L'Origine des espèces, William Worthington Fowler dans son classique Ten Years in Wall Street établit le premier parallèle entre Wall Street et la lutte pour la survie (1870:35).

\section{La langue de Wall Street utilisée dans la communication avec le grand public}

La langue que Wall Street utilise en interne est très différente de la langue à usage externe. Un exemple illustrera le contraste. Entre eux, les professionnels désignent volontiers les petits investisseurs par des appellations aux connotations méprisantes: outsiders, little guys, weak hands, Aunt Janes, widows and orphans, barefoot pilgrims, hogs, lame ducks, suckers, lambs, lobby rats (Odean 1988 : 46-51). Pourtant, l'activité boursière a besoin de leurs dollars et la langue externe de Wall Street oublie toute connotation négative à l'égard des non-professionnels et s'adapte à leurs attentes. Elle s'adresse à eux grâce à d'innombrables recommandations d'analystes, des articles de presse, des lettres circulaires boursières (stock market newsletters) ou des prospectus de premières mises sur le marché. Toutes ces publications sont actuellement complétées par des conseils en investissement et des courriels promotionnels envoyés gratuitement sur Internet. À cette longue liste, il faut ajouter The Exchange, la publication officielle du NYSE, désormais en ligne, qui communique avec les investisseurs ${ }^{2}$. 
La langue de Wall Street à destination du grand public évite les extrêmes du spectre terminologique et se concentre sur le groupe 3, mi-technique, mi-métaphorique. Les vulgarités argotiques en sont absentes de même que le vocabulaire trop technique des groupes 1 et 2. Le grand réseau métaphorique darwinien et son bestiaire en ont totalement disparu. Les professionnels de Wall Street masquent leurs rivalités et se présentent au public comme les artisans unis et responsables du miracle économique américain (Elias 1971 : 48, 178). La langue développe une rhétorique de la séduction visant à susciter l'enthousiasme des investisseurs. Les valeurs deviennent des growth/ performance stocks, des hot stocks, ou des wonder stocks. Les métaphores sont fréquemment sexuelles mais, afin d'éviter de choquer, elles restent dans le registre modéré (glamour stocks, go-go stocks). Les messages font une large place aux produits et aux technologies qui retiennent l'attention du public. Dans les années 1920, les seuls mots radio et airlines suffisaient pour tourner la tête aux investisseurs (Odean 1988 : 7, 175 ; Gordon 1999 : 227). Après le vol de Lindbergh à travers l'Atlantique en 1927, ils se précipitèrent aveuglément sur les titres des sociétés d'aviation, y compris sur ceux de la Seaboard Airlines Company, qui était en fait une société de chemin de fer (Odean 1988: 7). Dans les années 1990, les seuls mots « dot.com» ajoutés au nom des compagnies en faisaient flamber les cours. Une citation de Jack Dreyfus, qui dirigeait une firme de Wall Street dans les années 1960, montre par quels mécanismes lexicaux la rhétorique de la séduction fonctionne. Son efficacité repose sur le choix des mots les plus accrocheurs même si personne ne les comprend.

Take a nice little company that's been making shoelaces for 40 years and sells at a respectable six times earnings ratio. Change the name from Shoelaces Inc. to Electronics and Silicon Furth-Burners. In today's market, the words "electronics" and "silicon" are worth 15 times earnings. However, the real play comes from the word furth-burners, which no one understands. A word no one understands entitles you to double your entire score. Therefore we have six times earnings for the shoelace business and 15 times earnings for electronics and silicon, or a total of 21 times earnings. Multiply this by two for furth-burners and we now have a score of 42 times earnings for the new company. (Malkiel 1999: 58-59)

Des expressions martelées par les médias, comme « railroad boom, radio fever, petroleum frenzy, dot.com mania» ou "Internet craze» (Malkiel 1999: 90-94), visent à susciter des réactions grégaires chez des millions d'investisseurs qui ne veulent pas être laissés pour compte. Tocqueville note que les Américains sont sans cesse tourmentés par une crainte vague de n'avoir pas choisi la route la plus courte qui mène au bien-être (1981:171). Cette angoisse est entretenue par des messages tels que : "It's a once in a lifetime opportunity ", " Don't be left behind, invest in stocks». Des slogans comme "Own your share of American business », «Don't sell America short », "Shareownership, USA » (Elias 1971: 49) jouent sur le patriotisme des citoyens. Il leur est suggéré qu'investir en bourse est un acte de foi dans l'Amérique et que douter de Wall Street est « downright unAmerican ».

Pourtant, malgré leur assurance enthousiaste, les recommandations de Wall Street sont tempérées par de nombreuses précautions oratoires (hedging) qui multiplient les conditions (if), les restrictions (although) et les relativisations, comme par exemple: " Anyone can speculate [assurance enthousiaste] and, if he's willing to take the time and effort to study the situation [condition], he can be a successful speculator in the normal course of events [relativisation] ( Fuller 1962:212). Ou bien: «We believe it is reasonable to anticipate a priceearnings multiple of about 15 in a favorable stock market atmosphere " (Elias 1971:85). Ou encore : «I would hold the stock if I owned it, though I would not buy it » (Elias $1971: 87$ ). 
27 Aux précautions oratoires s'ajoutent les précautions légales fournies par l'anglais juridique. Depuis une vingtaine d'année, le legalese se répand dans le discours boursier, particulièrement dans les prospectus d'émissions de titres qui sont généralement rédigés par les avocats d'affaires de Wall Street. Au jargon financier se superposent les phrases longues et complexes des juristes. Leur vocabulaire opaque et le formatage compact des paragraphes souvent rédigés en lettres capitales en rendent la lecture difficile et empêchent les investisseurs de se faire une idée claire des bénéfices potentiels et des risques encourus. C'est la raison pour laquelle ces documents sont surnommés red herrings dans l'argot de Wall Street (Elias 1971 : 88). La couleur rouge vient de la bande écarlate qui en barre verticalement les pages. Quant aux harengs, ils étaient jadis jetés en pâture aux chiens pour les détourner au dernier moment du gibier qu'ils avaient pourchassé (Odean 1988 : 14). La métaphore laisse entendre que ces documents servent à attirer les investisseurs tout en les privant des profits qui reviendront aux initiés de Wall Street.

La rhétorique de la séduction, les précautions oratoires, l'opacité de l'anglais juridique alimentent l'idée que Wall Street use d'un double langage (double talk). Les exemples abondent où l'information enthousiaste (bullish) fournie aux investisseurs oublie de mentionner que l'entreprise émettrice ne fait aucun bénéfice ou perd de l'argent. Dans les années 1990, de nombreuses publications de résultats officieux (pro forma) laissaient entrevoir des gains mirifiques et poussaient le cours des actions vers les sommets, alors que les chiffres calculés selon les normes comptables officielles GAAP se soldaient par des pertes. Le double langage de certaines vedettes de Wall Street a été exposé sur la place publique en 2000 lorsque des analystes et des courtiers, comme Henry Blodget de chez Merrill Lynch, ont dû répondre de l'inculpation de mensonge. Ils recommandaient publiquement des titres à l'achat tout en conseillant en privé à leurs collègues de les vendre. Ces messages confidentiels étaient souvent accompagnés de commentaires péjoratifs, et même scatologiques, caractéristiques de l'argot de Wall Street: «That stock is such a piece of crap $»^{3}$. Ces violents contrastes linguistiques illustrent parfaitement l'existence des deux facettes distinctes, interne et externe, au sein de la langue de Wall Street et ils confirment l'hypothèse émise au terme de la première partie de cet article.

Le double langage de Wall Street, tel que le perçoit le grand public, a suscité de sa part de multiples réactions qui sont à l'origine des «Wall Street decoders». Il s'agit de lettres d'information, de sites sur Internet ou d'ouvrages, qui se sont spécialisés dans le décryptage des messages de la communauté boursière. Ainsi, le livre de David Caruso et de Robert Powell, Decoding Wall Street, promet d'expliquer "What the pros are saying - and what it really means». Pour sa part, le site Oak Ridger Online propose A Guide to Understanding Wall Street Lingo, et d'expliquer: "Wall Street has unique language where the meaning of the statement is different than the meaning of the words $»^{4}$.

Le double langage de Wall Street a suscité une telle dégradation de la confiance du public envers la communauté boursière à la fin des années 1990 que les autorités financières américaines ont dû réagir dans un domaine totalement inédit pour elles : le domaine linguistique. Dans un article précédent, nous proposions d'appeler « éléments-janus » les facteurs qui rééquilibrent l'asymétrie d'information entre Wall Street et Main Street (Van der Yeught 2003 : 7-9). À côté des janus informels, technologiques et organisationnels, nous plaçons les janus institutionnels comme la Securities and Exchange Commission (SEC). Créée dans les années 1930 à la suite du krach boursier de 1929, la SEC est garante de la qualité de l'information financière, et la loi de 1933 qu'elle doit faire appliquer est appelée le Truth in Securities Act. Les autorités de la SEC viennent donc d'enrichir son rôle 
de janus institutionnel en se rendant compte que lutter contre l'asymétrie d'information ne se limite pas à améliorer le contenu des messages. L'effort doit également porter sur la langue elle-même. Une campagne originale a été lancée en 1998 par le président de la SEC de l'époque, Arthur Levitt: The Plain English Initiative (Levitt 2002 : 157, 226, 245, 255). Cette campagne encourage Wall Street et les entreprises cotées à communiquer leurs informations en anglais simple et clair (plain English). Un Plain English Guide Book de 80 pages disponible en ligne ${ }^{5}$ explique comment éviter le jargon financier et l'opacité de la langue juridique. Par exemple, il conseille la voie active, qui identifie le sujet du verbe, et il déconseille la voie passive, qui le masque. Il recommande les phrases courtes, les présentations structurées, les textes en minuscules et non alignés à droite. Il donne des exemples de documents originaux, opaques avant traitement, et clarifiés après réécriture par les experts de la SEC. Un de ces experts se trouve être William D. Lutz, professeur d'anglais à l'université Rutgers et auteur de nombreux ouvrages sur le double langage.

L'initiative de la SEC est très originale car elle contraste fortement avec la tradition américaine de la norme impérative faible. P. Lerat rappelle que la norme impérative est l'obligation légale imposée par les autorités d'utiliser certains mots plutôt que d'autres (1983 : 23-24). Cette norme, assez contraignante en France et surtout au Québec, est singulièrement absente de la langue de Wall Street, le secteur boursier étant largement auto-réglementé aux États-Unis. Certes, le Plain English Guide Book n'impose rien de façon contraignante, mais il montre clairement que, pour lutter contre l'asymétrie d'information, la SEC a décidé d'assumer un rôle de janus linguistique.

\section{Conclusion}

32 En conclusion, la langue de Wall Street se présente historiquement comme la langue traditionnelle d'une communauté professionnelle ancienne, fermée et masculine, qui utilise un lexique motivé par l'usage. Cette langue soude les membres du milieu professionnel et comporte des marqueurs d'initiation qui permettent de repérer et d'exclure les non-initiés. Elle perçoit ses activités de façon largement métaphorique en adéquation avec les analyses de Lakoff et Johnson. Depuis les années 1970, cependant, Wall Street accueille de nouveaux métiers dont la terminologie est scientifiquement motivée. Sous leur influence, la langue originelle s'est enrichie d'une terminologie motivée qui respecte les exigences wüsteriennes et qui est largement symétrique avec les autres langues. Ces tendances opposées ont généré un spectre terminologique hétérogène. Cependant, ce langage ne facilite pas la communication avec le grand public américain car Wall Street l'utilise avec une certaine duplicité pour attirer les investisseurs tout en préservant ses privilèges. La récente Plain English Initiative de la SEC montre que la lutte contre l'asymétrie d'information passe également par un travail linguistique.

33 L'analyse de la langue de Wall Street qui vient d'être menée laisse présager quel peut être son avenir. C'est un lieu commun que de souligner le dynamisme de l'anglais de la bourse et de dire à quel point il influence les autres langues dans le contexte financier. De fait, il constitue l'une des facettes de l'impérialisme linguistique américain. Les autorités françaises, qui représentent la norme impérative en France, s'en inquiètent et recommandent dans leur Dictionnaire des termes officiels de la langue française (1994: 221-295), d'éviter, ou de remplacer par des mots français, de nombreux américanismes en provenance de Wall Street. À y regarder de près cependant, cette langue apparaît à la fois 
très forte et très fragile. Le lexique que Wall Street exporte dans le monde entier va de la terminologie motivée au lexique légèrement métaphorique (groupes 1 à 3). Ainsi, les Français en ont-ils largement adopté le jargon technique (swap, put, call, price/earnings ratio (PER), rating, alpha, back office, hedging, spread, spot). En revanche, ils ont négligé les termes métaphoriques des groupes 4 et 5 tels que bull, bear et blue chip qui sont pourtant très anciens. L'argot métaphorique est peu symétrique dans les autres langues. Cette partie traditionnelle de la langue de Wall Street est en réalité menacée. Elle dépend pour sa survie de la négociation à la criée et du contact direct entre les opérateurs du parquet. C'est dans la foule vociférante du trading floor que se forgent les métaphores, que jaillissent l'humour et les plaisanteries osées et que les codes boursiers sont sémantisés de façon souvent outrancière. Or, le parquet de New York est l'un des derniers du monde. Il survit aujourd'hui comme un anachronisme parmi des systèmes boursiers automatisés. Le jour où les ordinateurs remplaceront les hurlements des traders et où le silence envahira le parquet, le vieil argot de Wall Street entrera en agonie. La langue se recomposera dans le sens de la motivation. Elle gagnera sûrement en qualité terminologique, mais elle perdra aussi beaucoup en épaisseur humaine.

\section{BIBLIOGRAPHIE}

Beeman, Larry. 2001. A Guide to Understanding Wall Street Lingo. <http://www.oakridger.com/ stories/010801/bus_0108010024.html>.

Bernstein, Peter L. 1992. Capital Ideas : The Improbable Origins of Modern Wall Street. New York: The Free Press.

Caruso, David \& Robert Powel. 2001. Decoding Wall Street. New York : McGraw-Hill.

Colbert, David. 2001. Eye Witness to Wall Street. New York : Broadway Books.

Dandrey, Patrick. 1996 (Klincksieck 1991). Poétique de La Fontaine (1), La fabrique des fables, Paris : PUF Quadrige.

Dictionnaire des termes officiels de la langue française. 1994. Délégation générale à la langue française.

Elias, Christopher. 1971. Fleecing the Lambs: A Startling Look at Wall Street's Most Powerful InstitutionThe New York Stock Exchange. Chicago : Henry Regnery Company.

Fowler, W. Worthington. 1870. Ten Years in Wall Street, or Revelations of Inside Life and Experience on 'Change'. Hartford, Conn. : Worthington, Dustin \& Co.

Freud, Sigmund. 1930. Le Mot d'esprit et ses rapports avec l'inconscient. Paris : Gallimard Idées.

Fuller, John G. 1962. The Money Changers: The Story of the Insiders Who Really Run Wall Street. New York : The Dial Press.

Gordon, John Steele. 1999. The Great Game: A History of Wall Street. Londres : Orion Business Books.

Guilbert, L. 1973. «La Spécificité du terme scientifique et technique ». Langue française 17, 5-17.

Guiraud, Pierre. 1978. Les Mots savants. Paris : PUF. 
Hofstadter, Richard. 1959 [1944]. Social Darwinism in American Thought. New York : George Braziller, Inc.

Humbley, John. 2003. Applications de la terminologie. Paris 7. <http://rech.eila.jussieu.fr/ED/dea/ files/ApplicationsterminologieCF.rtf>.

Jacquillat, Bertrand \& Bruno Solnik. 1989. Marchés financiers : gestion de portefeuille et des risques. Paris : Dunod.

Lakoff, George \& Mark Johnson. 1980. Metaphors We Live By. Chicago : University of Chicago.

Lerat, Pierre. 1983. Sémantique descriptive. Paris : Hachette Université.

Lerat, Pierre. 1990. «L'Hypéronymie dans la structuration des terminologies ». Langage 98. Paris : Université Paris 13.

Lerat, Pierre. 1995. Les Langues spécialisées. Paris : PUF.

Lerat, Pierre. 1997. « Approches linguistiques des LSP ». ASp 15-18, 1-10.

Levison, Leonard Louis. 1961. Wall Street, a Pictorial History. New York : Ziff-Davis Publishing Company.

Levitt, Arthur. 2002. Take on the Street: What Wall Street and Corporate America Don't Want You to Know. New York : Pantheon Books.

Malkiel, Burton G. 1999. A Random Walk Down Wall Street. New York : W. W. Norton \& Company.

Medbury, James K. 1968 [1870. Boston, Mass. : Fields, Osgood \& Co.]. Men and Mysteries of Wall Street. Wells, Vermont : Fraser Publishing Company.

Ney, Richard. 1970. The Wall Street Jungle. New York : Grove Press.

Niederhoffer, Victor. 1997. The Education of a Speculator. New York : John Wiley \& Sons.

Odean, Kathleen. 1988. Wall Street Slang: High Steppers, Fallen Angels, \& Lollipops. New York : Dodd, Mead \& Company.

Partington, Alan. 1995. « A Corpus-based Investigation into the Use of Metaphor in British Business Journalism ». ASp 7-10, 25-39.

Pessin, Allan H. 1988. The Illustrated Encyclopaedia of the Securities Industry. New York : The New York Institute of Finance.

Resche, Catherine. 1995. « La langue de la Bourse : convergences et divergences ». ASp 7-10, 91-112.

Ricœur, Paul. 1975. La Métaphore vive. Paris : Seuil.

Setti, Skora. 2000. La Relation concept-objet autour des définitions de termes. Vienne (Autriche) : Termnet Publisher.

Shook, Robert J. \& Robert L. Shook. 1990. The Wall Street Dictionary. New York : New York Institute of Finance.

Sobel, Robert. 1970. The Curbstone Brokers. Washington, D.C. : Beard Books.

Sperandeo, Victor. 1993. Methods of a Wall Street Master. New York : John Wiley \& Sons.

Tocqueville, Alexis de. 1981 [1840]. De la démocratie en Amérique, tome 2. Paris : Garnier Flammarion.

Van Camperhoudt, Marc. 1997. Abrégé de terminologie multilingue. Centre de recherche $<$ Terminsti www.terminsti.refer.org>. 
Van der Yeught, Michel. 2003. «Le Transfert d'information, moteur de l'histoire boursière américaine ». ASp 39-40, 3-19.

Wall Street Joke Book. 1997. New York, N.Y. : St Martin's Press.

Zweig, Jason. n.d. « Wall Street Words ». Financial History 44, 32-33. New York : Financial History Museum.

\section{Sites Internet}

<http://www.stockfraudlawyernetwork.com/page1.php>.

<http://www.nyse.com/pdfs/xnlv11n05.pdf>.

<http://www.oakridger.com/stories/010801/bus_0108010024.html>.

$<$ http://sec.gov/pdf/handbook.pdf>.

\section{ANNEXES}

\section{Annexe}

\section{Tableau du spectre terminologique de la langue de Wall Street}

\begin{tabular}{|c|c|c|c|c|c|}
\hline \multicolumn{6}{|c|}{$\begin{array}{l}\text { Axe de la motivation des termes } \\
<=\text { Motivation forte }\end{array}$} \\
\hline & Groupe 1 & Groupe 2 & Groupe 3 & Groupe 4 & Groupe 5 \\
\hline $\begin{array}{l}\text { Degré de } \\
\text { motivation }\end{array}$ & $\begin{array}{l}\text { Motivation } \\
\text { totale }\end{array}$ & $\begin{array}{l}\text { Motivation } \\
\text { forte }\end{array}$ & $\begin{array}{l}\text { Motivation } \\
\text { moyenne }\end{array}$ & $\begin{array}{l}\text { Motivation } \\
\text { faible }\end{array}$ & $\begin{array}{l}\text { Motivation } \\
\text { nulle, } \\
\text { opacification }\end{array}$ \\
\hline Monosémie & Totale & Forte & Bonne & Bonne & Bonne \\
\hline $\begin{array}{l}\text { Approche } \\
\text { onomasiogique/ } \\
\text { sémasiologique }\end{array}$ & Onomasiologique & Onomasiologique & Mixte & Sémasiologique & Sémasiologique \\
\hline Dénotation & Totale & Forte & Forte & Forte & Forte \\
\hline Connotation & Nulle & Faible & $\begin{array}{l}\text { Moyenne, } \\
\text { variable }\end{array}$ & $\begin{array}{l}\text { Moyenne à } \\
\text { forte selon } \\
\text { contextes }\end{array}$ & $\begin{array}{l}\text { Moyenne à } \\
\text { forte selon } \\
\text { contextes }\end{array}$ \\
\hline Symétrie & Totale à forte & Bonne & $\begin{array}{l}\text { Moyenne, } \\
\text { variable }\end{array}$ & $\begin{array}{l}\text { Variable à } \\
\text { difficile }\end{array}$ & Très difficile \\
\hline $\begin{array}{l}\text { Transcription/ } \\
\text { recherche } \\
\text { d'équivalences }\end{array}$ & $\begin{array}{l}\text { Automatique, } \\
\text { facile }\end{array}$ & Facile & À adapter & $\begin{array}{l}\text { Variable à } \\
\text { difficile }\end{array}$ & Très difficile \\
\hline
\end{tabular}




\begin{tabular}{|c|c|c|c|c|c|}
\hline Classification & Facile & Facile & Variable & Difficile & Rare et difficile \\
\hline Normalisation & Facile & Facile & Difficile & $\begin{array}{l}\text { Difficile, } \\
\text { impossible }\end{array}$ & Impossible \\
\hline Exemples & $\begin{array}{l}\text { beta, alpha, } \\
\text { delta, PER }\end{array}$ & $\begin{array}{l} \\
\\
\text { stock / share, } \\
\text { bond, preferred } \\
\text { stock, voting } \\
\text { stock, swap, put, } \\
\text { call, spread, } \\
\text { margin, } \\
\text { prospectus, } \\
\text { primary / } \\
\text { secondary } \\
\text { market, warrant }\end{array}$ & $\begin{array}{l}\text { broker, } \\
\text { trader, } \\
\text { specialist, } \\
\text { hitech } \\
\text { stock, } \\
\text { penny } \\
\text { stock, } \\
\text { glamour } \\
\text { stock, } \\
\text { dot.com } \\
\text { stock, junk } \\
\text { bond, Wall } \\
\text { Street, } \\
\text { Black } \\
\text { Tuesday, } \\
\text { specialist, } \\
\text { stock split, } \\
\text { contrarian, } \\
\text { chinese } \\
\text { wall }\end{array}$ & $\begin{array}{l}\text { bear, bull, blue } \\
\text { chip, } \\
\text { bellwether, DK, } \\
\text { nifty-fifty, Big } \\
\text { Board, black } \\
\text { knight, poison } \\
\text { pill, Mayday, } \\
\text { fallen angel, } \\
\text { short, long, } \\
\text { vulture } \\
\text { investor, } \\
\text { crown jewels, } \\
\text { scalping, dawn } \\
\text { raid, bottom } \\
\text { fisher, } \\
\text { churning, road } \\
\text { show, sigles } \\
\text { sémantisés } \\
\text { identifiables }\end{array}$ & $\begin{array}{l}\text { triple-witching } \\
\text { hour, red } \\
\text { herring, blue- } \\
\text { sky laws, pink } \\
\text { sheets, sigles } \\
\text { sémantisés } \\
\text { cryptés (Clara } \\
\text { Bow) }\end{array}$ \\
\hline
\end{tabular}

\section{NOTES}

1. J'écris les mégamétaphores en majuscules selon l'usage de Lakoff \& Johnson (1980).

2. <http://www.nyse.com/pdfs/xnlv11n05.pdf>.

3. <http://www.stockfraudlawyernetwork.com/page1.php>.

4. <http://www.oakridger.com/stories/010801/bus_0108010024.html>.

5. <http://sec.gov/pdf/handbook.pdf>.

\section{RÉSUMÉS}

La langue des boursiers américains est à la fois technique et métaphorique. Elle sert une communauté financière jalouse de ses intérêts et lui permet de communiquer avec le grand public. Cet article se propose de montrer comment ces facettes opposées et leurs conflits d'intérêt sous-jacents se reflètent dans cette langue spécialisée. La première partie met en évidence l'hétérogénéité terminologique de la langue de Wall Street et exprime l'hypothèse qu'elle a des caractéristiques différentes selon que les professionnels communiquent entre eux ou s'adressent à des non-initiés. La deuxième partie analyse les discours «initiés-vers-initiés ». 
La troisième partie analyse les discours "initiés-vers-non-initiés » et montre qu'ils augmentent l'asymétrie d'information entre les initiés et les non-initiés au lieu de la réduire. La conclusion suggère que la langue traditionnelle et métaphorique de Wall Street laissera place à une terminologie plus scientifique sous l'effet de l'automatisation croissante de l'activité boursière et du décloisonnement du milieu professionnel.

The language used by Wall Street operators is both technical and metaphorical. It is used by a financial community which is keen to protect its interests and it is also a means of communicating with ordinary investors. This article shows how these contrasting aspects, and their underlying conflicts of interest, are reflected in this specialised language. The first part gives evidence that the language of Wall Street is terminologically heterogeneous and that it develops different styles depending on whether Wall Street insiders communicate with each other or with outsiders. The second part analyses "insider-to-insider" types of discourse. The third part analyses "insider-to-outsider" types of discourse and shows that they increase asymmetric information between insiders and outsiders instead of reducing it. Our conclusion suggests that a more scientific terminology will replace the traditional metaphorical facet of the language when computers replace the trading floor and as the barriers protecting insiders slowly crumble.

\section{INDEX}

Mots-clés : grand public, langue spécialisée, milieu professionnel, motivation, terminologie, Wall Street

Keywords : English for Specific Purposes, ESP, general public, motivation, professional environment, terminology, Wall Street

\section{AUTEUR}

\section{MICHEL VAN DER YEUGHT}

Michel Van der Yeught est maître de conférences au département GEA de l'IUT de Toulon. Il enseigne l'anglais de la finance et de la comptabilité et a choisi Wall Street comme thème de recherche (langue de spécialité et civilisation). mvdy@free.fr 